\title{
EDUCAÇÃO PARA SUSTENTABILIDADE E TEORIAS DE APRENDIZAGEM: UM ESTUDO BIBLIOMÉTRICO DOS ÚLTIMOS 10 ANOS
}

Data de submissão: 07/09/2017

Aceite: 31/07/2018

Nathália Rigui Trindade ${ }^{1}$

Carolina Sampaio Marques ${ }^{2}$

Maíra Nunes Piveta ${ }^{3}$

Rodrigo Reis Favarin ${ }^{4}$

Suelen Geíse Telocken ${ }^{5}$

Marcelo Trevisan ${ }^{6}$

\section{RESUMO}

Uma poderosa ferramenta de mudanças e transformações sociais para um futuro sustentável tem sido considerada a Educação para Sustentabilidade (EpS). Em vista disto, para que os resultados esperados e os objetivos da EpS sejam alcançados, novas propostas pedagógicas são necessárias, uma vez que a mudança de comportamentos e de atitudes deve ser alcançada a partir de abordagens pedagógicas que sejam centradas na criticidade dos sujeitos. Para que isso seja possível, a EpS encontra oportunidades nas teorias de aprendizagem de epistemologia construtivista, como: Teoria de Aprendizagem Experiencial, Aprendizagem Transformadora, Aprendizagem Libertadora e Aprendizagem Social. O objetivo deste estudo constitui-se em identificar e analisar as características das publicações científicas sobre o tema EpS vinculadas às teorias de aprendizagem Experiencial, Transformadora, Libertadora e Social. A operacionalização do levantamento deu-se por meio de um estudo bibliométrico, com buscas na Web of Science e Scopus nos últimos dez anos, e também, nos anais dos eventos da Associação Nacional de Pós-Graduação e Pesquisa em Administração (ANPAD). Os resultados evidenciam que essas são temáticas que estão em evidência nos últimos anos, sendo que a teoria da aprendizagem social é a que se encontra mais consolidada na literatura quando se trata de educação para sustentabilidade.

Palavras-chave: Educação para Sustentabilidade; Teorias de Aprendizagem; Pesquisa Bibliométrica.

\footnotetext{
1 Possui graduação em Administração pela Universidade Federal de Santa Maria, UFSM e mestrado em Administração pela UFSM. Santa maria - Rio Grande do Sul. Brasil. E-mail: nathaliariguitrindade@gmail.com

2 Possui graduação em Administração pela Universidade Federal de Santa Maria, UFSM, mestrado em Administração pela UFSM e doutorado em andamento em Administração pela UFSM. Santa maria - Rio Grande do Sul. Brasil. Email: marques_csm@yahoo.com.br 3 Possui graduação em Administração pela Universidade Federal de Santa Maria, UFSM, mestrado em Administração pela UFSM e doutorado em andamento em Administração pela UFSM. Santa maria - Rio Grande do Sul. Brasil. E-mail: mairanpiveta@gmail.com 4 Possui graduação em Administração pela Universidade Franciscana, UFN, mestrado em andamento em Administração pela Universidade Federal de Santa Maria, UFSM. Santa maria - Rio Grande do Sul. Brasil. E-mail: rodrigo.favarin@hotmail.com

5 Possui graduação em Administração pela Universidade de Cruz Alta, UNICRUZ, mestrado em Administração pela Universidade Federal de Santa Maria, UFSM. Santa maria - Rio Grande do Sul. Brasil. Email: stelocken@gmail.com

6 Possui graduação em Administração pela Universidade Federal de Santa Maria, UFSM, mestrado em Administração pela Universidade Federal de Santa Catarina, UFSC e doutorado em Administração pela Universidade Federal do Rio Grande do Sul, UFRGS. Santa Maria Rio Grande do Sul. Brasil Email: marcelotrev@gmail.com
} 


\section{INTRODUÇÃO}

É eminente a necessidade de mudanças e de transformações nos comportamentos da sociedade para que o desequilíbrio existente entre as esferas sociais, ambientais e econômicas seja superado, tornando efetivo o conceito de desenvolvimento sustentável. Muito se discute a respeito do papel da educação como um instrumento de legitimação perante a sociedade, de ações efetivas para a transformação positiva da realidade e o alcance de um futuro sustentável (JACOBI, 2005; ROWE, 2007; STERLING, 2011; CARS; WEST, 2014). Conforme a Organização das Nações Unidas para a Educação, a Ciência e a Cultura (UNESCO, 1997), embora não seja a resposta absoluta, a educação é parte vital dos esforços para o desenvolvimento de novas relações entre as pessoas e maior respeito às necessidades do meio ambiente.

Ademais, Sterling (2011) evidencia o paradoxo existente entre o poder de transformação da educação e a falta de correlação entre o alto desempenho educacional e comportamentos socialmente e ambientalmente sustentáveis. O que, conforme Sterling (2011), tem feito com que educadores interessados em sustentabilidade e justiça social passem a buscar nas teorias de aprendizagem possíveis caminhos para seguir. Isso corrobora a afirmação de Morin (2000), de que a educação no futuro deverá integrar os conhecimentos de maneira que estes sejam multidisciplinares, transversais, multidimensionais, transnacionais, globais e planetários, evidenciando a necessidade de uma renovação nos modelos pedagógicos atuais.

Diante disso, conforme apontam Jacobi, Raufflet e Arruda (2011), a educação para sustentabilidade exige novas propostas pedagógicas que sejam "centradas na criticidade dos sujeitos, com vistas à mudança de comportamento e atitudes, ao desenvolvimento da organização social e da participação coletiva" (JACOBI; RAUFFLET; ARRUDA, 2011, p. 28). Deste modo, entende-se que é necessária a transição de um modelo de aprendizagem baseado no professor e no conteúdo para uma abordagem centrada no aluno.

O cerne deste processo de mudança está na busca e na integração de diferentes teorias de aprendizagem relacionadas à atividade pedagógica; é preciso integrar a perspectiva construtivista, que vê o aluno como agente na construção do seu próprio conhecimento, sendo capaz de interpretar e representar criativamente o mundo e não apenas responder a ele (MOREIRA, 1999). Conforme defende Figueiró (2015), é preciso dar ênfase a situações de aprendizagem ativas, experienciais, colaborativas e dirigidas, possibilitando a solução dos problemas locais, regionais e globais.

Neste sentido emergem abordagens como a Teoria de Aprendizagem Experiencial, de David Kolb, a Teoria de Aprendizagem Transformadora, de Mezirow, a Teoria de Aprendizagem Libertadora, de Paulo Freire e a Teoria da Aprendizagem Social, tendo seu representante principal Albert Bandura, como algumas possibilidades de conceber e praticar formas educacionais que favoreçam a criação de condições que permitam o desenvolvimento sustentável. Vale destacar que, existem outras abordagens ligadas ao construtivismo, como as teorias de Vigotsky, Ausubel, Johnson-Laird, entre outras (MOREIRA, 1999). No entanto, neste estudo o foco será nas quatro teorias previamente apresentadas, pois ao consultar especialistas na área de educação para sustentabilidade, eles indicaram estas abordagens como as que mais se relacionam com a Educação para Sustentabilidade (EpS).

Tendo em vista o panorama apresentado, torna-se interessante analisar o seguinte problema de pesquisa: como se caracterizam a produção científica nacional e internacional sobre educação para sustentabilidade vinculada às teorias de aprendizagem experiencial, transformadora, libertadora e social? Para tanto, o objetivo da presente pesquisa constitui-se em identificar 
e analisar as características das publicações científicas sobre o tema educação para sustentabilidade vinculada às teorias de aprendizagem experiencial, transformadora, libertadora e social.

A partir disso, busca-se apresentar evidências, levantando informações e características para obter conhecimento das publicações referentes à temática. Ao considerar a complexidade da educação para a sustentabilidade, que abrange todas as áreas do conhecimento e não somente as ciências educativas, os resultados deste estudo podem auxiliar na ampliação do conhecimento a respeito de como as teorias de aprendizagem podem interferir no alcance dos objetivos da Educação para Sustentabilidade (EpS), tendo em vista que nem todos os interessados em EpS possuem formação em educação. Ademais, os insights deste estudo podem beneficiar uma série de processos institucionais, desde a concepção e revisão de programas acadêmicos (em especial novos programas emergentes), através de avaliações de ensino e aprendizagem.

A fim de responder ao questionamento, teve-se como referência os artigos publicados nos últimos dez anos, compreendendo o período de 2007 a 2016. Realizaram-se as buscas nas bases de dados Web of Science (WoS) e Scopus, além da pesquisa realizada nos anais dos eventos da Associação Nacional dos Programas de Pós-Graduação em Administração (ANPAD).

\section{EDUCAÇÃO PARA SUSTENTABILIDADE E TEORIAS DE APRENDIZAGEM}

As discussões a respeito da educação como uma ferramenta essencial para o desenvolvimento sustentável já são disseminadas por diversos órgãos e instituições, evidenciando a importância de uma educação de qualidade de forma integrada e interdisciplinar (UNESCO, 2012). Assim, a educação para o desenvolvimento sustentável é voltada à reorientação da educação em escala global que, conforme delineado no Capítulo 36 da Agenda 21 busca: reorientar a educação no sentido do desenvolvimento sustentável; aumentar a consciência pública; e promover treinamento para desenvolver recursos humanos para facilitar a transição para um mundo sustentável (BARBIERI; SILVA, 2011).

As Nações Unidas proclamaram a Década da Educação para o Desenvolvimento Sustentável (2005-2014), cujo objetivo global consiste em "integrar os valores inerentes ao desenvolvimento sustentável em todos os aspectos da aprendizagem com o intuito de fomentar mudanças de comportamento que permitam criar uma sociedade sustentável e mais justa para todos" (DESD, 2005, p.16). Com isso, a ONU busca desenvolver o pensamento crítico e resolutivo da sociedade com o intuito de propor questionamentos sobre o contexto atual e as possibilidades existentes para melhorar as relações entre os aspectos social, ambiental e econômico da comunidade que estamos inseridos (DESD, 2005).

Nesse sentido, Jacobi, Raufflet e Arruda (2011) afirmam que as ações de educação para sustentabilidade devem se basear em abordagens pedagógicas que objetivem a criticidade, a mudança de atitudes e comportamentos, a participação de toda a sociedade e o desenvolvimento de organizações sociais. Para que esses objetivos sejam alcançados, é necessário pensar e fazer a educação de uma maneira diferente do que é realizado hoje. A educação para sustentabilidade exige novos métodos e abordagens de ensino e de aprendizagem, é preciso pensar tanto no que se ensina, quanto como isto é realizado, assim, cada vez mais formas inovadoras de desenvolver as capacidades dos alunos devem ser buscadas (BRUNDIERS et al, 2010; ROWE, 2007).

Rowe (2007) afirma que a sustentabilidade precisa ser o foco de nossos esforços na educação. Conforme Sipos et. al (2008), a educação para a sustentabilidade deve estar preparada 
para desconstruir e reconstruir todos os aspectos do ensino e da aprendizagem. Segundo os autores, é possível mudar os padrões educacionais, que são voltados para a insustentabilidade - crises sociais e ecológicas - ao direcionar a abordagem pedagógica para o ensino da sustentabilidade - justiça social e ecológica. Neste contexto, ir além da reprodução de nossos problemas sociais requer transformação para novas formas de abordar a educação e a vida, o que exige inovações pedagógicas que possibilitem a aprendizagem interativa, experimental e transformadora do mundo real (SIPOS et al, 2008; BRUNDIERS et al, 2010; SIVAPALAN et al, 2015). Em seu estudo, Brundiers e Wiek (2013), afirmam que na literatura recente as abordagens de aprendizagem contextuais, construtivistas e colaborativas sobre o desenvolvimento de possíveis soluções para problemas de sustentabilidade do mundo real, envolvendo as partes interessadas, são as mais indicadas para a capacitação dos alunos em matéria de sustentabilidade.

A partir do Relatório de Monitoramento e Avaliação da Década de Educação para o Desenvolvimento Sustentável (DESD), Tilbury (2011), ao analisar e documentar diversos estudos, mostra que os processos de aprendizagem ativos e participativos estão alinhados com as intenções e as estruturas, e são percebidos como os mais apropriados para a aprendizagem da educação e para o desenvolvimento sustentável. Com isso, a aprendizagem ativa e participativa incentiva os alunos a: i) fazer perguntas reflexivas e críticas; ii) esclarecer valores; iii) projetar futuro mais positivo; iv) pensar sistematicamente; v) responder através da aplicação da aprendizagem e; vi) explorar a dialética entre tradição e inovação, o que faz com que estas sejam comumente consideradas um dos principais processos subjacentes à educação para o desenvolvimento sustentável (TILBURY, 2011).

Por isso, Sivapalan et. al (2015) coloca que as noções pedagógicas em torno do desenvolvimento sustentável são abordagens de ensino e de aprendizagem associadas à teoria do construtivismo. Isto porque o construtivismo vê o aluno como agente na construção do seu próprio conhecimento, sendo capaz de interpretar e representar criativamente o mundo, e não apenas responder a ele (MOREIRA, 1999). Ou seja, a ênfase é dada ao envolvimento e interação dos alunos, bem como entre alunos e professores, em uma comunidade de aprendizagem que promove a centralização, a reflexividade e a transformação do aluno (SIVAPALAN et. al, 2015).

Da perspectiva do construtivismo, emergem teorias como a Teoria de Aprendizagem Experiencial, de David Kolb, a Teoria de Aprendizagem Transformadora, de Mezirow, a Teoria de Aprendizagem Libertadora, de Paulo Freire e a Teoria da Aprendizagem Social, tendo seu representante principal Albert Bandura, como possibilidades de conceber e praticar formas educacionais que possibilitem a criação de condições que permitam o desenvolvimento sustentável. Baseado em Moreira (1999), vale destacar que neste trabalho o termo "teoria de aprendizagem" é usado sem muito rigor, sendo que uma teoria de aprendizagem é uma construção humana para interpretar sistematicamente a área do conhecimento, representando um ponto de vista de um autor/pesquisador sobre como interpretar o tema aprendizagem, o que é, como funciona e porque funciona (MOREIRA, 1999).

A teoria da aprendizagem experiencial de David Kolb (1984) origina-se com os trabalhos de Lewin, Dewey e Piaget, e consiste em uma abordagem interdisciplinar, holística e integradora da aprendizagem, que combina experiência, percepção, cognição e comportamento (KOLB; KOLB, 2008). Kolb (1984) destaca, em sua teoria, a importância da experiência, no sentido de experiência vivencial, para o desenvolvimento da aprendizagem e do conhecimento. Kolb (1984) define a aprendizagem experiencial como o processo pelo qual o conhecimento é criado por meio da transformação da experiência e resume os fundamentos da teoria em seis principais características: 1) a aprendizagem é mais bem concebida como um processo, e não em termos 
de resultados; 2) todo o aprendizado é um reaprendizado; 3) a aprendizagem requer a resolução de conflitos entre modos de adaptação ao mundo dialeticamente opostos; 4) aprendizagem é um processo holístico de adaptação; 5) aprendizagem envolve transações sinérgicas entre as pessoas e o meio ambiente e; 6) aprendizagem é o processo de criar conhecimento.

No que diz respeito à teoria de aprendizagem transformadora, esta surgiu a partir dos estudos de Mezirow (1978). A aprendizagem transformadora consiste no processo pelo qual se realiza a mudança em nossos quadros de referências, tornando-os mais inclusivos, abertos, emocionalmente capazes de mudanças e reflexivos (MEZIROW, 1997). Quadros de referências são estruturas de pressupostos através dos quais entendemos nossas experiências. Conforme Mezirow (1997), para transformar nossos quadros de referência é necessária a reflexão crítica sobre os pressupostos em que nossas crenças, interpretações e pontos de vista estão baseados. Sterling (2011) afirma que a aprendizagem transformadora se refere a uma mudança qualitativa na percepção e na criação de sentido por parte do aprendiz, em uma determinada experiência de aprendizagem, de tal forma que o aprendiz questiona ou reformula suas suposições ou hábitos de pensamento.

Sterling (2011) afirma também que a visão de Mezirow sobre o aprendizado transformador tem alguma ressonância com o conceito de conscientização de Freire (1972), que tem sido muito influente no discurso da pedagogia crítica e nos círculos de educação emancipatória. Conforme Maciel (2011), Paulo Freire foi um educador e militante que dedicou sua vida à construção de uma educação libertadora, produtora do diálogo permanente, a qual parte da problematização da realidade dos educandos para a finalidade de intervenção no mundo. Ainda, conforme Petroni e Souza (2009), dentro da aprendizagem libertadora, para Freire, entende-se o conceito de autonomia como algo resultante do desenvolvimento do sujeito, relacionado ao fato de ele tornarse capaz de resolver questões por si mesmo, de tomar decisões sempre de maneira consciente e estar pronto para assumir uma maior responsabilidade e assim arcar com as consequências de seus atos. Ademais, de acordo com Freire (1996, 1999, 2005 apud PETRONI; SOUZA, 2009), esse modelo de educação proporciona a aquisição de conhecimentos que respeitam a individualidade e que levam em consideração os conhecimentos prévios do aluno.

Finalmente, a teoria da aprendizagem social surgiu em 1970, quando Albert Bandura buscava descrever como os indivíduos aprendem e alteram seus comportamentos em um contexto social. De acordo com Bandura (1977), pode-se explicar o comportamento humano por meio das influências das estruturas comportamentais, cognitivas e ambientais. Ou seja, a aprendizagem dos indivíduos se dá por meio da interação ou da observação junto ao seu contexto social (FIGUEIRÓ, 2015). A aprendizagem social ao longo dos anos passou a ser estudada em diversas áreas, inclusive ligada à gestão de recursos naturais e à resolução de problemas complexos, casos característicos da sustentabilidade (HARMONICOP, 2005; GLASSER, 2007; KILVINGTON, 2010). O conceito de aprendizagem social expande o significado individualista da educação, no qual cada processo de aprendizagem é influenciado pelas interações sociais dos participantes e com o ambiente social, de maneira que soluções em comum acordo sejam alcançadas e que as circunstâncias do contexto sejam consideradas (DLOUHÁ et al, 2013). Para D'angelo e Brustein (2014), embora a teoria de Bandura (1977) seja desenvolvida para explicar o comportamento individual, ela também pode ser aplicada na investigação de como a aprendizagem coletiva ocorre. 


\section{MÉTODO}

Com o objetivo de identificar e analisar as características das publicações científicas sobre o tema educação para sustentabilidade, vinculada às teorias de aprendizagem experiencial, transformadora, libertadora e social, desenvolveu-se o presente estudo a partir de uma pesquisa bibliométrica. A bibliometria, técnica escolhida para este estudo, se dedica a quantificar, identificar, analisar e descrever uma série de padrões na produção de conhecimento científico sobre um tema específico (ARAÚJO, 2006).

No que diz respeito à abordagem da análise de dados, a presente pesquisa é de cunho quantitativo, caracterizada pela sistemática dos dados, manuseio de ferramentas estatísticas e por analisar um tema específico sem enfoque nas questões relativas à qualidade das publicações (MEADOWS, 1999; GREGOLIN et al., 2005).

\subsection{Coleta de dados}

Para a coleta de dados, utilizaram-se as bases de dados Web of Science (WoS) do Institute for ScientificInformation (ISI) e a Scopus, por estas apresentarem maior abrangência sobre o tema e oferecerem ferramentas bibliométricas úteis para a consolidação da pesquisa, bem como os anais dos eventos da Associação Nacional dos Programas de Pós-Graduação em Administração (ANPAD), devido a sua importância no cenário acadêmico nacional brasileiro.

Vale destacar que a Web of Science (WoS) constitui-se de uma base multidisciplinar, que indexa apenas periódicos com grande número de citações na web, oferecendo informações sobre o impacto e a visibilidade das publicações científicas, onde se pode analisar a produção científica com cálculos de índices bibliométricos e o percentual de autocitações, assim como a criação de rankings por inúmeros parâmetros (CAPES, 2017).

Em suma, a coleta das informações utilizadas nesta pesquisa foi realizada por meio do sistema Web of Science (WoS), Scopuse anais dos eventos da Associação Nacional dos Programas de Pós-Graduação em Administração(ANPAD). A realização da pesquisa dividiu-se em três etapas, conforme a Figura 1.

Figura 1 - Etapas da pesquisa

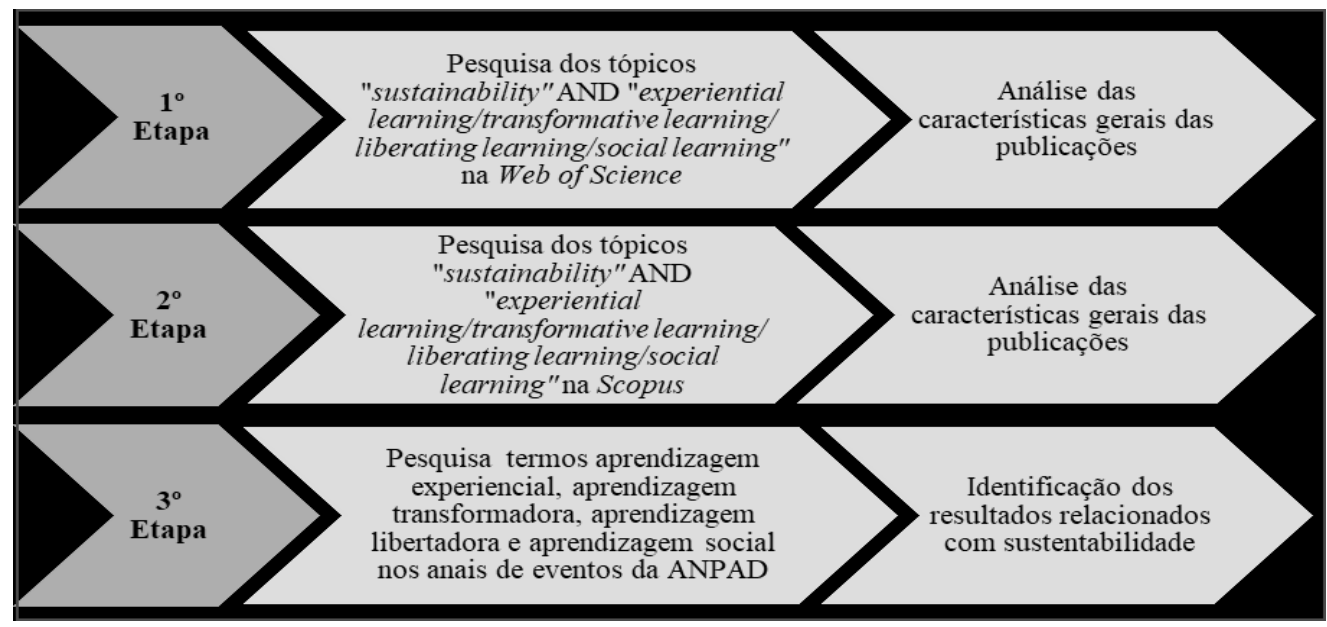

Fonte: Elaborado pelos autores. 
Na primeira etapa por meio do mecanismo de busca da Web of Science (WoS), realizouse a pesquisa pelo em tópico pelos termos "sustainability" AND "experientiallearning", em seguida "sustainability" AND "transformativelearning", posteriormente "sustainability" AND "liberatinglearning/education" e, por fim, buscaram-se os termos "sustainability" AND "social learning", delimitando todas as buscas para um período de dez anos, de 2007 a 2016, da Principal Coleção do Web of Science (WoS).

Cabe ressaltar que na busca por "sustainability" AND "liberatinglearning", realizouse também a pesquisa por "sustainability" AND "liberatingeducation", visando uma maior abrangência de busca, tendo em vista que, na primeira tentativa não se obteve respostas. Ademais, é importante destacar ainda, que se optou por utilizar o termo sustainability ao invés de education for sustainability, visando encontrar um maior número de trabalhos sobre o tema. Assim, na segunda etapa da pesquisa, repetiram-se exatamente os mesmos procedimentos na base de dados Scopus.

Por fim, na terceira etapa buscaram-se dados a respeito da publicação científica nacional no que tange à educação para sustentabilidade vinculada às teorias de aprendizagem. A operacionalização desta etapa deu-se por meio de levantamentos nos anais dos eventos da Associação Nacional dos Programas de Pós-Graduação em Administração (ANPAD). No campo de busca disponível no portal da ANPAD, inicialmente inseriu-se o termo aprendizagem experiencial, na sequência aprendizagem transformadora, após, aprendizagem libertadora (também se buscou por educação libertadora e pedagogia libertadora) e, por fim, buscou-se por aprendizagem social. Com os resultados da busca, realizou-se uma seleção, por meio da leitura dos títulos e resumos disponíveis, das publicações que vinculavam as teorias em questão com a sustentabilidade.

\subsection{Análise dos dados}

Para a análise dos dados da pesquisa nas bases de dados Web of Science (WoS) e Scopus foram utilizados os recursos de análise da WoS que geram gráficos de porcentagem, tendo como base o modelo conceitual que busca identificar as categorias dispostas no Quadro 1.

Ademais, para análise dos dados referentes ao levantamento nos anais dos eventos da Associação Nacional dos Programas de Pós-Graduação em Administração (ANPAD), características como título, autores, evento e ano de publicação foram utilizadas. De acordo com as etapas evidenciadas na Figura 1, realizou-se a análise bibliométrica proposta no presente estudo, cujos resultados serão apresentados na próxima seção.

Quadro 1 - Modelo conceitual para análise bibliométrica

\begin{tabular}{|c|c|}
\hline \multicolumn{2}{|c|}{ Características gerais das publicações } \\
\hline Total de publicações & Título das fontes \\
\hline Áreas temáticas & Anos de publicação \\
\hline Tipos de documentos & Idiomas \\
\hline Autores & Países \\
\hline
\end{tabular}

Fonte: elaborado pelos autores.

\section{APRESENTAÇÃO E ANÁLISE DOS RESULTADOS}

Nesta seção, os resultados das buscas realizadas sobre a temática sustentabilidade e teorias de aprendizagem, realizadas nas bases de dados Web of Science (WoS) eScopus, bem 


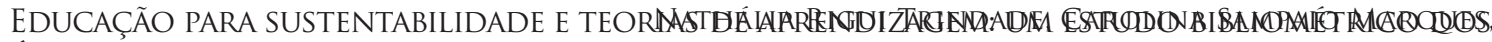
ÚLTIMOS 10 ANOS

como os da busca nos anais dos eventos da Associação Nacional dos Programas de Pós-Graduação em Administração (ANPAD), serão apresentados.

\subsection{Características Gerais das publicações nas bases Web of Science (WoS)e Scopus}

Na primeira fase da pesquisa, realizam-se a inserção dos tópicos "sustainability" AND "experientiallearning", em seguida "sustainability" AND "transformativelearning", posteriormente "sustainability" AND "liberatingeducation (learning)"e, por fim, os termos "sustainability" AND "social learning", inicialmente na base de dados Web of Science e, posteriormente, na Scopus. O número de registros encontrados para cada busca é demonstrado na Tabela 1.

Tabela 1 - Total de publicações

\begin{tabular}{|c|c|c|c|c|}
\hline \multirow{2}{*}{ Teorias de aprendizagem } & \multicolumn{2}{|l|}{ WoS } & \multicolumn{2}{|c|}{ Scopus } \\
\hline & Registros & $\%$ & Registros & $\%$ \\
\hline Teoria de aprendizagem experiencial (experientiallearning) & 78 & $25 \%$ & 144 & $29 \%$ \\
\hline Teoria de aprendizagem transformadora (transformativelearning) & 47 & $15 \%$ & 78 & $16 \%$ \\
\hline Teoria de aprendizagem libertadora (liberatingeducation/learning) & 0 & $0 \%$ & 0 & $0 \%$ \\
\hline Teoria de aprendizagem social (social learning) & 189 & $60 \%$ & 267 & $55 \%$ \\
\hline TOTAL & 314 & $100 \%$ & 489 & $100 \%$ \\
\hline
\end{tabular}

Fonte: elaborado pelos autores com base na Web of Science (WoS) e Scopus.

A partir da Tabela 1, é possível perceber que a maior parte das publicações que vinculam sustentabilidade às teorias de aprendizagem, tem como base a teoria de aprendizagem social, que apresentou 189 publicações como resultado da busca na WoS e 267 registros na Scopus. Já, dentre as teorias pesquisadas, a teoria de aprendizagem experiencial é a segunda com maior número de registros, apresentando 78 publicações na WoS e 144 na Scopus.

A partir desses resultados observados, a aprendizagem social tem se caracterizado como principal teoria de aprendizagem vinculada à temática sustentabilidade, e isso pode estar ligado ao fato de que o lócus desta abordagem está situado nas relações sociais e não em uma abordagem cognitivista, desta maneira o conhecimento e a realidade são constituídos por meio de interações sociais, seja pelo desenvolvimento de práticas ou pelas experiências conjuntas por parte dos atores envolvidos (BOUWEN E TAILLIEU, 2004), o que vai ao encontro da proposta da Educação para Sustentabilidade (EpS).

Somando-se a isso, é possível perceber que diversos estudos defendem que a aprendizagem experiencial é uma das formas mais eficazes de promover mudanças positivas em indivíduos e organizações (SVOBODA; WHALEN, 2004; BADEN; PARKES, 2013; ALVES; TOMETICH, 2016; UNESCO, 2017; CORSCADDEN; KEVANY, 2017), pois essa aprendizagem envolve o indivíduo por completo no processo, assim como a sua inteligência mental, emocional e somática, tratando as pessoas como um sistema vivo complexo, no qual "o aprendizado experiencial pode ser o tipo de experiência de aprendizado mais ecológico, além da própria experiência da vida real" (SVOBODA; WHALEN, 2004, p.172).

Vale destacar ainda que não foram encontrados resultados, em nenhuma das bases, para a busca pelos termos "sustainability" AND "liberatingeducation", nem mesmo "sustainability" AND "liberatinglearning".

Sendo assim, a seguir serão apresentadas as características gerais das publicações relacionadas ao tema de acordo com as seguintes categorias: autores, países, tipos de documentos, 
idiomas, ano das publicações, áreas temáticas e título das fontes, conforme exposto previamente no Quadro 1.

Os principais autores que publicaram a respeito da temática pesquisada são apresentados na Tabela 2.

Tabela 2 - Principais autores

\begin{tabular}{|c|c|c|c|c|}
\hline \multirow{3}{*}{ Teorias de aprendizagem } & \multicolumn{4}{|c|}{ Principais autores } \\
\hline & \multicolumn{2}{|c|}{ WoS } & \multicolumn{2}{|l|}{ Scopus } \\
\hline & Autor & Registro & Autor & Registro \\
\hline \multirow{4}{*}{$\begin{array}{l}\text { Teoria de aprendizagem experiencial } \\
\text { (experientiallearning) }\end{array}$} & WIEK, A. & 3 & LANDIS, A.E. & 5 \\
\hline & SEAGER, T. P. & 3 & BILEC, M.M. & 4 \\
\hline & LANDIS, A.E. & 3 & ANTAYA, C.L. & 3 \\
\hline & ANTAYA, C.L. & 3 & WIEK, A. & 3 \\
\hline \multirow{3}{*}{$\begin{array}{l}\text { Teoria de aprendizagem transformadora } \\
\text { (transformativelearning) }\end{array}$} & SINCLAIR, A.J. & 11 & SINCLAIR, A.J. & 12 \\
\hline & MOYER, J.M. & 3 & MOYER, J.M. & 4 \\
\hline & STERLING, $\mathrm{S}$. & 3 & STERLING, S. & 3 \\
\hline \multirow{2}{*}{$\begin{array}{l}\text { Teoria de aprendizagem libertadora } \\
\text { (liberatingeducation/learning) }\end{array}$} & - & - & - & - \\
\hline & \begin{tabular}{|c|}
- \\
- \\
-
\end{tabular} & - & - & $\overline{-}$ \\
\hline \multirow{3}{*}{ Teoria de aprendizagem social (social learning) } & ANGELSTAM, P. & 6 & ANGELSTAM, P. & 6 \\
\hline & AXELSSON, R. & 6 & AXELSSON, R. & 6 \\
\hline & ELBAKIDZE, $\mathrm{M}$. & 6 & ELBAKIDZE, $\mathrm{M}$. & 6 \\
\hline
\end{tabular}

Fonte: elaborado pelos autores com base na Web of Science e Scopus.

Observa-se, na Tabela 2, que nas duas bases de dados em que se realizaram as buscas, os autores destacados são comuns. Assim, para aquelas publicações que vinculam sustentabilidade com aprendizagem experiencial, pode-se destacar o autor LANDIS, A. E., já no que diz respeito à combinação de sustentabilidade com a teoria de aprendizagem transformadora, os resultados indicam que o autor que mais publicou sobre a temática é SINCLAIR, A.J.. E, por fim, ao relacionar sustentabilidade com aprendizagem social, os três autores que se destacam com a mesma quantidade de publicações são ANGELSTAM, P., AXELSSON, R. e ELBAKIDZE, M..

Em relação aos países que concentram as publicações sobre os assuntos, optou-se por selecionar os três mais representativos de cada um, conforme dados coletados na Web of Science (WoS) e na Scopus. Para a busca por "sustainability" AND "experientiallearning", os principais países que publicam sobre o assunto são Estados Unidos, Reino Unido e Canadá. No que tange à aprendizagem transformadora, destacam-se Austrália, Canadá e Estados Unidos. Por fim, quando combinado com a aprendizagem social, Reino Unido, Estados Unidos e Austrália se destacam. Além disso, ressalta-se que o Brasil não está presente entre os principais países que publicam sobre as temáticas citadas anteriormente, o que representa uma grande oportunidade de estudo para os pesquisadores brasileiros.

No que tange os tipos de documentos encontrados com a pesquisa nas bases de dados Web of Science (WoS) e Scopus, os resultados podem ser visualizados nas Tabelas 3 e 4. 
Tabela 3 - Principais tipos de documentos na Web of Science (WoS)

\begin{tabular}{|c|c|c|c|c|c|c|}
\hline \multicolumn{7}{|l|}{ WoS } \\
\hline \multirow[b]{2}{*}{ Teorias de aprendizagem } & \multicolumn{6}{|c|}{ Tipos de documentos } \\
\hline & Article & ProceedingsPaper & Review & $\begin{array}{l}\text { Editorial } \\
\text { Material }\end{array}$ & $\begin{array}{l}B \text { o } \\
\text { Chapter }\end{array}$ & Total \\
\hline $\begin{array}{l}\text { Teoria de aprendizagem } \\
\text { experiencial (experientiallearning) }\end{array}$ & 56 & 22 & - & - & - & 78 \\
\hline $\begin{array}{l}\text { Teoria de aprendizagem } \\
t \mathrm{r} \text { a } \mathrm{n} \mathrm{s} \mathrm{f} \text { o } \mathrm{r} \mathrm{m} \text { a d o } \mathrm{r} \text { a } \\
\text { (transformativelearning) }\end{array}$ & 38 & 6 & 2 & 1 & - & 47 \\
\hline $\begin{array}{l}\text { Teoria de aprendizagem libertadora } \\
\text { (liberatingeducation/learning) }\end{array}$ & - & - & - & - & - & - \\
\hline $\begin{array}{l}\text { Teoria de aprendizagem social } \\
\text { (social learning) }\end{array}$ & 170 & 10 & 5 & 3 & 1 & 189 \\
\hline Total & 264 & 38 & 7 & 4 & 1 & 314 \\
\hline
\end{tabular}

Fonte: elaborado pelos autores com base na Web of Science(WoS).

Tabela 4 - Tipo de documento Scopus

\begin{tabular}{|c|c|c|c|c|c|c|c|c|c|c|c|}
\hline \multicolumn{12}{|c|}{ Scopus } \\
\hline \multirow[b]{2}{*}{$\begin{array}{c}\text { Teorias de } \\
\text { aprendizagem }\end{array}$} & \multicolumn{11}{|c|}{ Tipos de documentos } \\
\hline & Article & $\begin{array}{l}\text { Conferen- } \\
\text { cePaper }\end{array}$ & Review & $\begin{array}{c}\text { Article } \\
\text { Press }\end{array}$ & $\begin{array}{c}\text { Conference- } \\
\text { Review }\end{array}$ & $\begin{array}{c}\text { Book } \\
\text { Chapter }\end{array}$ & Book & Editorial & Note & $\begin{array}{l}\text { Short } \\
\text { Survey }\end{array}$ & Total \\
\hline $\begin{array}{c}\text { Teoria de } \\
\text { aprendizagem } \\
\text { experiencial } \\
\text { (experiential- } \\
\text { learning) }\end{array}$ & 95 & 30 & 8 & 2 & 4 & 4 & 1 & - & - & - & 144 \\
\hline $\begin{array}{c}\text { Teoria de } \\
\text { aprendizagem } \\
\text { transforma- } \\
\text { dora (trans- } \\
\text { formativelear- } \\
\text { ning) }\end{array}$ & 55 & 9 & 5 & 3 & - & 5 & 1 & - & - & - & 78 \\
\hline $\begin{array}{c}\text { Teoria de } \\
\text { aprendizagem } \\
\text { libertadora } \\
\text { (liberatin- } \\
\text { geducation/ } \\
\text { learning?) }\end{array}$ & - & - & - & - & - & - & - & - & - & - & - \\
\hline $\begin{array}{c}\text { Teoria de } \\
\text { aprendizagem } \\
\text { social (social } \\
\text { learning) }\end{array}$ & 191 & 16 & 18 & 6 & 1 & 26 & 5 & 2 & 1 & 1 & 267 \\
\hline Total & 341 & 55 & 31 & 11 & 5 & 35 & 7 & 2 & 1 & 1 & 489 \\
\hline
\end{tabular}

Fonte: elaborado pelos autores com base na Scopus.

De acordo com as Tabelas 3 e 4, no que tange aos tipos de documentos, pode-se averiguar que na Web of Science (WoS) e na Scopus, a predominância de documentos deu-se no formato de artigo, em todas as buscas realizadas, independentemente da teoria de aprendizagem vinculada. Ademais, outro formato de documento com maior número de registros consiste em artigos publicados em eventos. 
Destaca-se, ainda, que a teoria da aprendizagem social foi a única que se encontrou em todos os tipos de documentos pesquisados, dentro das bases Web of Science (WoS) e Scopus, o que pode evidenciar a importância e sua vasta aplicabilidade dentro de contextos sociais (DLOUHÁ et al, 2013).

A seguir, na Tabela 5, apresentam-se os principais idiomas das publicações encontradas na base Web of Science (WoS), para cada tipo de busca. Vale destacar que, essa informação não estava disponível para a base de dados da Scopus no momento da realização da pesquisa.

Tabela 5 - Principais idiomas

\begin{tabular}{c|c|c|c|c}
\hline & \multicolumn{5}{c}{ WoS } \\
\cline { 2 - 5 } Teorias de aprendizagem & Inglês & Espanholis idiomas & Alemão & Russo \\
\hline $\begin{array}{c}\text { Teoria de aprendizagem experiencial } \\
\text { (experientiallearning) }\end{array}$ & 74 & 2 & - & - \\
\hline $\begin{array}{c}\text { Teoria de aprendizagem transformadora } \\
\text { (transformativelearning) }\end{array}$ & 46 & - & 1 & - \\
\hline $\begin{array}{c}\text { Teoria de aprendizagem libertadora } \\
\text { (liberatingeducation/learning) }\end{array}$ & - & - & - & - \\
\hline $\begin{array}{c}\text { Teoria de aprendizagem social } \\
\text { (sociallearning) }\end{array}$ & 187 & - & 1 & 1 \\
\hline
\end{tabular}

Fonte: elaborado pelos autores com base na Web of Science (WoS).

A partir da análise da Tabela 5, é possível notar que, no que diz respeito aos principais idiomas em que os trabalhos são publicados, há uma grande predominância da língua inglesa e as demais línguas aparecem com menor representatividade, já que não possuem diferenças significativas de representatividade, sendo elas: espanhol (aprendizagem experiencial), alemão (aprendizagem transformadora e social) e russo (aprendizagem social).

As Figuras 1 e 2 abordam a relação da quantidade de documentos no que tange à distribuição entre os anos que a presente pesquisa abordou.

Figura 2 - Anos de publicação da revista Web of Science (WoS)

\section{Anos de Publicação (WoS)}

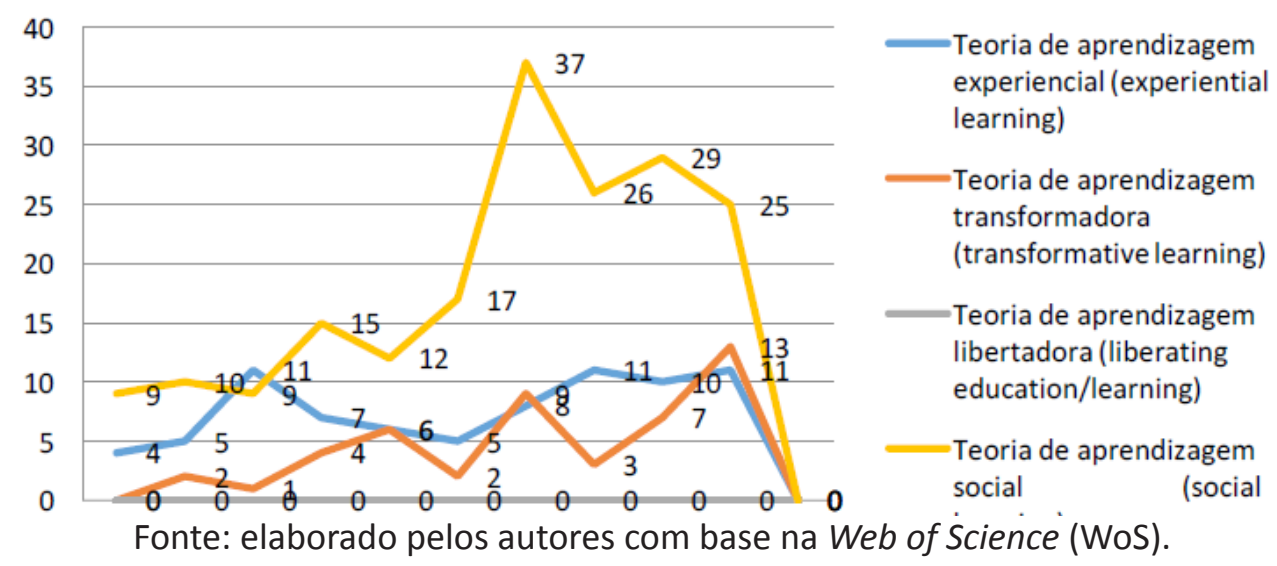


Figura 3 - Anos de publicação da revista Scopus

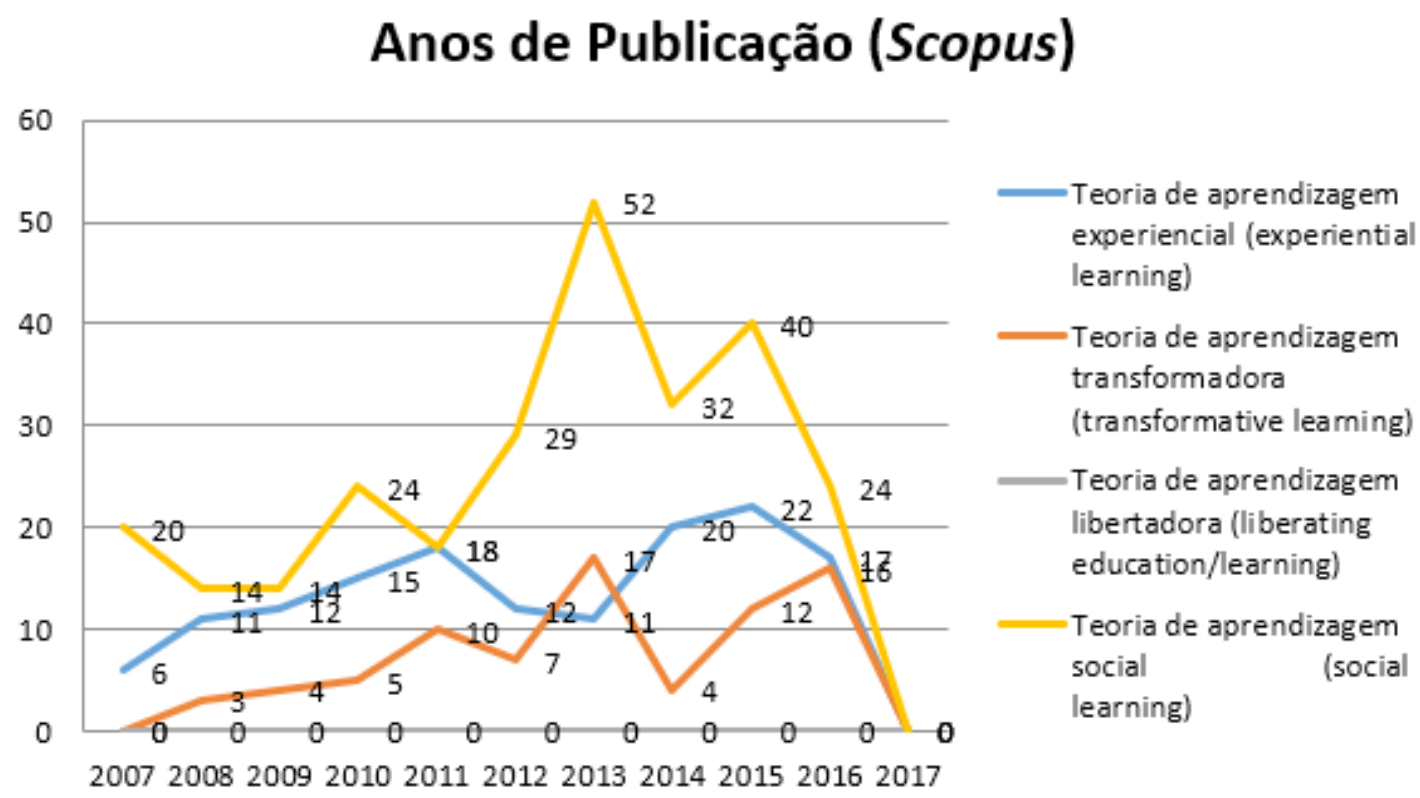

Fonte: elaborado pelos autores com base na Scopus.

Como pode ser visto a partir das figuras, os anos que apresentaram mais documentos a respeito da temática sustentabilidade e aprendizagem experiencial foram os de 2014, 2015 e 2016, demonstrando o crescente desenvolvimento de estudos sobre a temática nos últimos anos. Já, em relação aos estudos sobre sustentabilidade e aprendizagem transformadora, é possível notar que os anos 2013 e 2016 se destacaram em publicações sobre o assunto, não havendo uma constância de publicações sobre o tema. Por fim, no que tange à sustentabilidade e a aprendizagem social, o ano em que mais se publicou sobre o assunto foi o de 2013, com uma queda no número de publicações nos últimos dois anos.

Outro aspecto categórico averiguado consistiu nos títulos das fontes, disponíveis na base de dados da revista Web of Science (WoS). Os resultados obtidos para a busca de sustentabilidade e aprendizagem experiencial indicam que as duas fontes com maior número de registro são International Journal of Sustainability in Higher Education (5 registros) e A see Annual Conference Exposition (4 registros). Já, no que tange à sustentabilidade e aprendizagem transformadora, as fontes que se destacam são intituladas International Journal of Sustainability in Higher Education (4 registros) e Environmental Education Research (4 registros).E, quando se trata de sustentabilidade e aprendizagem social, Ecology And Society (11 registros) e Ecological Economics (9 registros) são os títulos da fonte que mais apresentam registros.

Por fim, nas Tabelas 6 e 7, as quatro principais áreas de pesquisa, da base Web of Science (WoS) e Scopus, respectivamente, são apresentadas e destacadas. 
Tabela 6 - Áreas de pesquisa - WoS

\begin{tabular}{c|c|c|c|c|c}
\hline & \multicolumn{5}{|c}{ WoS } \\
\cline { 2 - 6 } Teorias de aprendizagem & $\begin{array}{c}\text { EducationEducational- } \\
\text { Research }\end{array}$ & $\begin{array}{c}\text { Environmental } \\
\text { SciencesEcology }\end{array}$ & Engineering & $\begin{array}{c}\text { Science } \\
\text { Technology } \\
\text { OtherTopics }\end{array}$ & $\begin{array}{c}\text { Business } \\
\text { Economics }\end{array}$ \\
\hline $\begin{array}{c}\text { Teoria de aprendizagem } \\
\text { experiencial } \\
\text { (experientiallearning) }\end{array}$ & 26 & 21 & 18 & 16 & 7 \\
\hline $\begin{array}{c}\text { Teoria de aprendizagem } \\
\text { transformadora } \\
\text { (transformativelearning) }\end{array}$ & 23 & 19 & 2 & 11 & 3 \\
\hline $\begin{array}{c}\text { Teoria de aprendizagem } \\
\text { libertadora } \\
\text { (liberatingeducation/ } \\
\text { learning) }\end{array}$ & - & 120 & 18 & - & - \\
\hline $\begin{array}{c}\text { Teoria de aprendizagem } \\
\text { social (sociallearning) }\end{array}$ & 20 & - & 35 & 23 \\
\hline
\end{tabular}

Fonte: elaborado pelos autores com base na Web of Science(WoS).

Por meio da Tabela 6, pode-se constatar que, segundo dados da Web of Science(WoS), as áreas temáticas Education Educational Research,Environmental Sciences Ecology, Engineering e Science Technology Other Topics se destacaram na busca por sustentabilidade e aprendizagem experiencial, com ênfase para a primeira. Quando se trata de sustentabilidade e aprendizagem transformadora, as mesmas áreas temáticas se destacaram, com exceção da Engineering. Na sequência, ao analisar sustentabilidade vinculada à aprendizagem social, as áreas de pesquisa em destaque são Environmental SciencesEcology, Science Technology OtherTopics, Business Economicse EducationEducationalResearch, sendo a primeira a mais expressiva.

Tabela 7 - Áreas de pesquisa - Scopus

\begin{tabular}{c|c|c|c|c|c|c}
\hline \multirow{2}{*}{ Teorias de aprendizagem } & \multicolumn{7}{c}{ Scopus } \\
\cline { 2 - 7 } & $\begin{array}{c}\text { Social } \\
\text { Sciences }\end{array}$ & $\begin{array}{c}\text { Environmental } \\
\text { Science }\end{array}$ & $\begin{array}{c}\text { Business, } \\
\text { Management } \\
\text { and Acco... }\end{array}$ & Engineering & Energy & $\begin{array}{c}\text { Agricultural } \\
\text { And } \\
\text { Biological } \\
\text { Sciences }\end{array}$ \\
\hline $\begin{array}{c}\text { Teoria de aprendizagem } \\
\text { experiencial (experiential } \\
\text { learning) }\end{array}$ & 79 & 27 & 26 & 26 & 19 & $\mathbf{5}$ \\
\hline $\begin{array}{c}\text { Teoria de aprendizagem } \\
\text { transformadora } \\
\text { (transformative learning) }\end{array}$ & 54 & 23 & 13 & 7 & 8 & 6 \\
\hline $\begin{array}{c}\text { Teoria de aprendizagem } \\
\text { libertadora (liberating } \\
\text { education/learning) }\end{array}$ & - & - & - & - & - & - \\
\hline $\begin{array}{c}\text { Teoria de aprendizagem social } \\
\text { (social learning) }\end{array}$ & 127 & 136 & 42 & 29 & 26 & 38 \\
\hline
\end{tabular}

Fonte: elaborado pelos autores com base na Scopus. 
Ao analisar a Tabela 7, é possível evidenciar as principais áreas de pesquisa para cada busca na base de dados da revista Scopus. Assim, no que tange à sustentabilidade e a aprendizagem experiencial, as áreas de maior destaque são Social Sciences, Environmental Science, Business, Management and Accountability e Engineering. Em relação à sustentabilidade e aprendizagem transformadora, destacam-se áreas como Social Sciences, Environmental Science, Business, Management and Accountability e Energy. E no que tange à aprendizagem social, o destaque vai para Environmental Science, seguida de Social Sciences, Business, Management and Accountability e Agricultural and Biological Sciences.

Conforme apresentado nas Tabelas 6 e 7, torna-se possível verificar a quantidade de publicações que tratam da aprendizagem social dentro da área de Environmental Sciences, o que demonstra a relação inicial do uso da teoria da aprendizagem social com a gestão de recursos naturais, como é exposto no referencial teórico (HARMONICOP, 2005; GLASSER, 2007; KILVINGTON, 2010). Além disso, a utilização da aprendizagem experiencial em ciências sociais pode estar atrelada ao fato de que diversos autores classificam a aprendizagem experiencial como um componente-chave no processo de integração da sustentabilidade ao ensino (WRIGHT, 2006; DIELEMAN; HUISINGH, 2006; DOMASK, 2007; BADEN; PARKES, 2013; ALVES; TOMETICH, 2016; CORSCADDEN; KEVANY, 2017) sendo, também, bastante mencionada em estudos sobre o processo de ensino aprendizagem nos cursos de gestão e negócios (CORSCADDEN; KEVANY, 2017).

\subsection{Levantamento nos anais da Associação Nacional de Pós-Graduação e Pesquisa em Adminis- tração(ANPAD)}

A presente seção destina-se a apresentar o contexto nacional a respeito das publicações sobre a temática da sustentabilidade e as teorias de aprendizagem selecionadas. Para tanto, realizaramse levantamentos nos anais dos eventos promovidos pela Associação Nacional de Pós-Graduação e Pesquisa em Administração (ANPAD) em um espaço de tempo que compreendeu os últimos dez anos, em decorrência da relevância da Associação no cenário acadêmico nacional da Administração.

Esta etapa da pesquisa foi realizada ao buscar-se pelos termos "aprendizagem experiencial", na sequência "aprendizagem transformadora", após "aprendizagem libertadora/ educação libertadora/ pedagogia libertadora" e, por fim, buscou-se por "aprendizagem social" no campo de busca do portal ANPAD. Os resultados obtidos com a pesquisa estão dispostos na Tabela 8.

Tabela 8 - Resultados busca ANPAD

\begin{tabular}{c|c|c}
\hline \multirow{2}{*}{ Teorias de aprendizagem } & $\begin{array}{c}\text { Total de } \\
\text { Registros }\end{array}$ & $\begin{array}{c}\text { ANPAD } \\
\text { Relacionados com } \\
\text { Sustentabilidade }\end{array}$ \\
\hline Aprendizagem experiencial & 5 & 1 \\
\hline Aprendizagem transformadora & 5 & 2 \\
\hline Aprendizagem libertadora & 0 & 0 \\
\hline Aprendizagem social & 9 & 5 \\
\hline TOTAL & $\mathbf{1 9}$ & $\mathbf{8}$
\end{tabular}

Fonte: elaborado pelos autores com base no portal ANPAD.

Como é possível notar, a partir da Tabela 8, a busca que mais obteve resultados, assim como nos resultados obtidos com a Web of Science (WoS)e Scopus, foi a relacionada à aprendizagem social, onde obteve-se 9 publicações relacionadas com o assunto, sendo que 5 delas estão ligadas ao tema sustentabilidade. Com mesma representatividade, na busca inicial, a aprendizagem experiencial e transformadora apresentaram no total 5 publicações cada uma, 
sendo que a aprendizagem transformadora apresentou uma publicação a mais vinculada ao tema sustentabilidade, totalizando 2 publicações sobre a temática-alvo desta pesquisa.

Cabe destacar que, assim como nos resultados obtidos na busca por aprendizagem libertadora na Web of Science (WoS) e Scopus, não foi possível obter resultados para esta teoria. Visando ampliar a busca, inseriu-se, além do termo "aprendizagem libertadora", os termos "educação libertadora" e "pedagogia libertadora", que também não apresentaram resultados. Na sequência, serão apresentadas as características das publicações (título, autores, evento e ano) encontradas que vinculam as teorias de aprendizagem à sustentabilidade. O Quadro 2 evidencia os resultados para a aprendizagem experiencial.

Quadro 2 - Trabalhos nos anais dos eventos da ANPAD

\begin{tabular}{|c|c|c|c|}
\hline \multicolumn{4}{|c|}{ Aprendizagem experiencial } \\
\hline Título & Autores & Evento & Ano \\
\hline $\begin{array}{c}\text { Teoria da Aprendizagem Experiencial e } \\
\text { Design Thinking para Criação de uma Feira de } \\
\text { Sustentabilidade }\end{array}$ & Nilo Barcelos Alves; PatriciaTometich & EnANPAD & 2016 \\
\hline \multicolumn{4}{|c|}{ Aprendizagem transformadora } \\
\hline Título & Autores & Evento & Ano \\
\hline $\begin{array}{c}\text { Framework da Aprendizagem Transformadora } \\
\text { Sustentável: integrando os Processos de } \\
\text { Ensino-Aprendizagem, Gestão e Mudança para } \\
\text { Sustentabilidade }\end{array}$ & $\begin{array}{c}\text { Lisiane Celia Palma; Eugenio } \\
\text { AvilaPedrozo }\end{array}$ & EnANPAD & 2015 \\
\hline $\begin{array}{l}\text { Reflexão Crítica e Aprendizagem } \\
\text { Transformadora: mensurando a Racionalidade } \\
\text { de Valor Compartilhado no Ensino de Estratégia } \\
\text { para Sustentabilidade }\end{array}$ & $\begin{array}{c}\text { JanetteBrunstein; Marta Fabiano } \\
\text { Sambiase; Marcos Bidart Carneiro de } \\
\text { Novaes }\end{array}$ & EnANPAD & 2015 \\
\hline \multicolumn{4}{|c|}{ Aprendizagem social } \\
\hline Título & Autores & Evento & Ano \\
\hline $\begin{array}{l}\text { A Emergência da Aprendizagem Social no } \\
\text { Ambiente de Gestão das Águas de Rios }\end{array}$ & $\begin{array}{c}\text { Lúcia Rejane da Rosa Gama Madruga; } \\
\text { Tania Nunes da Silva; Lucas Veiga } \\
\text { Ávila }\end{array}$ & EnANPAD & 2015 \\
\hline $\begin{array}{l}\text { Aprendizagem Social para Sustentabilidade: } \\
\text { um Estudo em um Curso de Administração de } \\
\text { Empresas }\end{array}$ & $\begin{array}{l}\text { Andreza Sampaio de Mello; Arilda } \\
\text { Schmidt Godoy }\end{array}$ & EnANPAD & 2015 \\
\hline $\begin{array}{l}\text { Aprendizagem Social Organizacional e } \\
\text { Sustentabilidade: a Experiência de um Programa } \\
\text { Empresarial de Mulheres Empreendedoras }\end{array}$ & Diego Gama Amaral; JanetteBrunstein & EnANPAD & 2015 \\
\hline $\begin{array}{c}\text { O Processo de Aprendizagem Social nos } \\
\text { Comitês ou Conselhos Voltados às Questões de } \\
\text { Desenvolvimento Sustentável }\end{array}$ & $\begin{array}{l}\text { Marcia Juliana d'Angelo; } \\
\text { JanetteBrunstein }\end{array}$ & EnEO & 2014 \\
\hline $\begin{array}{c}\text { Aprendizagem Social para a Sustentabilidade: } \\
\text { um Estudo Sobre Negócios Sustentáveis em } \\
\text { Contextos de Múltiplos Atores Sociais, Relações } \\
\text { e Interesses }\end{array}$ & $\begin{array}{l}\text { Marcia Juliana d'Angelo; } \\
\text { JanetteBrunstein }\end{array}$ & EnANPAD & 2013 \\
\hline
\end{tabular}

Fonte: elaborado pelos autores com base no portal da Associação Nacional de Pós-Graduação e Pesquisa em Administração (ANPAD). 
Como pode ser visualizado no Quadro 2, foram encontrados na totalidade 8 trabalhos publicados nos anais dos eventos da ANPAD que abordassem ao menos uma das teorias de aprendizagem e sustentabilidade nos últimos dez anos. O evento EnANPAD apresentou a maioria (7) dos trabalhos em seus anais com a temática nos anos de 2013, 2014 e 2016, enquanto que o EnEO apresentou um trabalho no ano de 2014. É válido destacar que o tema-alvo deste estudo tem demonstrado um crescente número de publicações no cenário nacional nos últimos anos. O maior número de trabalhos encontrados referentes a teoria da aprendizagem social, pode vir a exemplificar a sua possibilidade de aplicação tanto com o foco no comportamento individual, como também, ao investigar a aprendizagem coletiva (D’ANGELO; BRUSTEIN, 2014).

\section{CONSIDERAÇÕES FINAIS}

A partir da observância acerca da importância da educação na sociedade e da, cada vez mais urgente, necessidade de se pensar e agir pela sustentabilidade e pelo desenvolvimento sustentável, o objetivo deste estudo foi identificar e analisar as características das publicações científicas sobre o tema educação para sustentabilidade vinculada às teorias de aprendizagem experiencial, transformadora, libertadora e social. Assim, foi possível levantar informações e características para obter conhecimento das publicações referentes ao assunto.

Predominantemente, o que se observou por meio da realização do estudo bibliométrico está de acordo com o que afirma Sterling (2004, p.50) de que o desenvolvimento sustentável fornece "uma porta de entrada para uma visão diferente da pedagogia", tendo em vista os resultados encontrados para cada uma das buscas realizadas. No entanto, é possível perceber que essas são temáticas que estão em evidência nos últimos anos, sendo que a teoria da aprendizagem social é a que se encontra mais consolidada na literatura quando se trata de educação para sustentabilidade.

Em relação aos resultados obtidos nas bases Web of Science (WoS) e Scopus, tem-se o resultado de que a proeminente maioria das publicações consiste em artigos, publicados em grande parte nos anos de 2013 a 2016, sendo que a fonte de publicação com maior destaque foi a International Journal of Sustainability in Higher Education. No que tange aos países de origem, os Estados Unidos, Canadá, Reino Unido e Austrália figuraram como os mais relevantes em termos de quantidade de publicação nos últimos dez anos. Por fim, a língua inglesa destacou-se como o idioma mais utilizado nas publicações acerca dos temas. No cenário nacional também foi possível encontrar resultados para as buscas que demonstram o crescimento das publicações sobre o assunto nos últimos anos.

Ademais, o presente estudo evidenciou oportunidades de pesquisa no campo de Educação para Sustentabilidade (EpS) vinculada às teorias de aprendizagem, tendo em vista o crescente enfoque nas diferentes abordagens pedagógicas para o desenvolvimento sustentável. Dessa forma, ressalta-se a necessidade de continuidade e aprofundamento nos estudos sobre o assunto e a realização de um estudo qualitativo a respeito destas publicações seria interessante.

Finalmente, as limitações do presente estudo residem, primeiramente, na ausência de um rigor metodológico no que se refere às viabilizações das etapas de pesquisa desenvolvidas no método de busca. E, segundamente, na forma com que se procedeu as buscas neste estudo, levando em conta uma compilação de diversos empregos metodológicos encontrados em vários estudos bibliométricos, o que acarretou na impossibilidade da generalização dos resultados. 


\section{REFERÊNCIAS}

ALVES, N. B.; TOMETICH, P. Teoria da Aprendizagem Experiencial e Design Thinking para Criação de uma Feira de Sustentabilidade. In: XL Encontro da ANPAD - EnANPAD, 2016.

ARAÚJO, C.A. Bibliometria: Evolução Histórica e Questões Atuais. Em Questão, Porto Alegre, v. 12, n. 1, p. 11- 32, 2006.

BADEN, D.; PARKES, C. Experiential learning: inspiring the business leaders of tomorrow. Journal of Management Development, v. 32, n.3, p. 295-308, 2013.

BANDURA A. Social learningtheory. EnglewoodCliffs: Prentice Hall, 1977.

BARBIERI, J. C. e SILVA D. Desenvolvimento sustentável e educação ambiental: uma trajetória comum com muitos desafios. Revista ADM. MACKENZIE, V. 12, N. 3, maio/jun. 2011.

BOUWEN, R.; TAILLIEU, T. Multi-partyCollaboration as Social Learning for Interdependence: DevelopingRelationalKnowing for Sustainable Natural Resource Management. JournalofCommunity\&Applied Social Psychology, v. 14, n. 3, p. 137-153, 2004.

BRUNDIERS, K.; WIEK, A. Do We Teach What We Preach? An International Comparison of Problem- and Project-Based Learning Courses in Sustainability. Sustainability. v.5, p.1725-1746, 2013.

BRUNDIERS, K.; WIEK, A.; REDMAN, C.L. Real-world learning opportunities in sustainability: from classroom into the real world. International Journal of Sustainability in Higher Education. v. 11, n.4, 2010.

CARS, M.; WEST, E. E. Education for sustainable society: attainments and good practices in Sweden during the United Nations Decade for Education for Sustainable Development (UNDESD). Environment, Development and Sustainability, 2014.

CORSCADDEN, K. W.; KEVANY, K. The TREEhouse: A hybrid model for experiential learning in environmental education. Applied Environmental Education\& Communication, feb., 2017.

D'ANGELO, M, J.; BRUNSTEIN, J. Social learning for sustainability: supporting sustainable business in Brazil regarding multiple social actors, relationships and interests. International Journal of Sustainable Development \&World Ecology, v. 21, n.3, p. 273-289, 2014.

DLOUHÁ, J., BARTON, A., JANOUŠKOVÁ, S., \& DLOUHÝ, J. Social learning indicators in sustainability-oriented regional learning networks. Journal of Cleaner Production, v. 49, p. 6473, 2013.

TILBURY, D. Década da Educação das Nações Unidas para um Desenvolvimento Sustentável, 2005-2014: documento final do esquema internacional de implementação. - Brasília, 2005. 
DIELEMAN, H.; HUISINGH, D. Games by which to learn and teach about sustainable development: exploring the relevance of games and experiential learning for sustainability. Journal of Cleaner Production, v.14, p.837-847, 2006.

DOMASK, J. J. Achieving goals in higher education. An experiential approach to sustainability studies. International Journal of Sustainability in Higher Education, v. 8, iss 1, p. 53 - 68, 2007.

FIGUEIRÓ, P. S. Educação para a Sustentabilidade em cursos de graduação em Administração: Proposta de uma estrutura analítica. Tese de doutorado. Programa de Pós-Graduação em Administração da Universidade Federal do Rio Grande do Sul, Porto Alegre, 2015.

FREIRE, P. Pedagogy of the Oppressed. Harmondsworth: Penguin, 1972.

GLASSER, H. Minding the gap: the role of social learning in linking our stated desire for a more sustainable world to our everyday actions and policies. In: WALS, A. E. J. Social learning: towards a sustainable world. Wageningen: Wageningen Academic Publishers, 2007. p. 35-61.

GREGOLIN, J. A. R.; et al. Análise da produção cientifica a partir de indicadores bibliométricos. In: FUNDAÇÃO DE AMPARO À PESQUISA DO ESTADO DE SÃO PAULO. Indicadores de ciência, tecnologia e inovação em São Paulo 2004. São Paulo: FAPESP, 2005.

HARMONISING COLLABORATIVE PLANNING, HARMONICOP. Learning together to manage together: improving participation in water management, Osnabrück, 2005.

JACOBI, P. R. Educação ambiental: o desafio da construção de um pensamento crítico, complexo e reflexivo. Educ. Pesqui. [online]. vol.31, n.2, pp. 233-250, 2005.

JACOBI, P.R.; RAUFFLET, E.; ARRUDA, M. P. de. Educação para a sustentabilidade nos cursos de Administração: Reflexão sobre paradigmas e práticas. RAM, Rev. Adm. Macke, 2011.

KILVINGTON, M. Building Capacity for Social Learning in Environmental Management. 2010. Tese (Doutorado em Filosofia). Lincoln University, Canterbury, Nova Zelândia, 2010.

KOLB, A.; KOLB, D. Experiential learning theory: a dynamic, holistic approach to management learning, education and development, Handbook of Management Learning, Education and Development,2008.

KOLB, D. A. Experiential learning: experience as the source of learning and development. Englewood Cliffs, NJ: Prentice Hall, 1984.

MACIEL, K. de F. O pensamento de Paulo Freire na trajetória da educação popular. Educação em Perspectiva, Viçosa, v. 2, n. 2, p. 326-344, jul./dez. 2011.

MEADOWS, A. J. A Comunicação Científica. Brasília: Briquet de Lemos, 1999.

MEZIROW, J. Transformative Learning: Theory to Practice. New Directions for Adult And continuing Education, n.74, Summer 1997. 
MOREIRA, M.A. Teorias de aprendizagem. São Paulo: Editora Pedagógica e Universitária Ltda, 1999.

MORIN, E. Os sete saberes necessários à educação do futuro. Edgar Morin: tradução de Catarina Eleonora F. da Silva e Jeanne Sawaya ; revisão técnica de Edgard de Assis Carvalho. - 2. ed. - São Paulo : Cortez; Brasília, DF : UNESCO, 2000.

PETRONI, A.P.; SOUZA, V. L. T. de. VIGOTSKI E PAULO FREIRE: contribuições para a autonomia do professor. Rev. Diálogo Educ., Curitiba, v. 9, n. 27, p. 351-361, maio/ago. 2009.

ROWE, D. Education for a sustainable future. Science, p.317-323, 2007.

SVOBODA, S.; WHALEN, J. Using experiential simulation to teach sustainability. In Galea, C. Ed. Teaching business sustainability: cases, simulations and experimental approaches. Greenleaf Publishing Limited: Sheffield. p. 171-179, 2004.

SIPOS, Y.; BATTISTI, B.; GRIMM, K. Achieving transformative sustainability learning: engaging heads, hands and heart. International Journal of Sustainability in Higher Education. v. 9, n.1, p. 68-86, 2008.

SIVAPALAN, S.; SUBRAMANIAM, G.; CLIFFORD, M. J. Institutional Practices Versus Student Needs and Its Implications for the Development of a Holistic Engineering Education for Sustainable Development (EESD) Framework. Transformative Approaches to Sustainable Development at Universities. Springer, p.413-433, 2015.

STERLING, S. Higher Education, Sustainability and the Role of Systemic Learning, in Higher Education and The Challenge of Sustainability, op. cit., p.49-70, 2004.

STERLING, S. Transformative Learning and Sustainability: sketching the conceptual ground. Learning and Teaching in Higher Education, Issue 5, 2011.

TILBURY, D.: An expert review of processes and learning. Paris: UNESCO, 2011.

UNESCO. Educación para un futuro sostenible: una visión transdisciplinaria para una acción concertada. Paris: Unesco, EPD-97/CONF.401/CLD.1, 1997.

UNESCO. Teaching and Learning for a Sustainable Future: a multimedia teacher education programme. Disponível em: < http://www.unesco.org/education/tlsf/index.html >. Acesso em: fevereiro de 2017.

UNESCO, Shaping the education of tomorrow. Report on the UN decade of education for sustainable development. Abridged, Paris, 2012.

WRIGHT, T. S. A. Feeling Green: Linking Experiential Learning and University Environmental Education. Higher Education Perspectives, 2(1), p.73-90, 2006. 